\title{
Characteristics of carbapenemase-producing Klebsiella pneumoniae as a cause of neonatal infection in Shandong, China
}

\author{
YAN JIN $^{1 *}$, XIAOFEI SONG ${ }^{2 *}$, YIGANG LIU $^{1}$, YONG WANG $^{1}$, \\ BINGCHANG ZHANG ${ }^{1}$, HUI FAN ${ }^{1}$ and CHUNHONG SHAO ${ }^{1}$ \\ Departments of ${ }^{1}$ Microbiology and ${ }^{2}$ Immunology, Clinical Laboratory, \\ Provincial Hospital Affiliated to Shandong University, Jinan, Shandong 250021, P.R. China
}

Received May 8, 2015; Accepted September 1, 2016

DOI: $10.3892 /$ etm.2017.4070

\begin{abstract}
The goal of the present study was to examine the characteristics of carbapenem-resistant Klebsiella pneumoniae as a cause of neonatal infection. A total of 37 carbapenem-resistant Klebsiella pneumoniae-positive newborns hospitalized in Shandong Provincial Hospital, China between April 2011 and October 2013 were examined. Antibiotic susceptibility testing was performed using the agar dilution method and the Etest. Resistance genes were assessed by polymerase chain reaction (PCR) and sequencing. Pulsed-field gel electrophoresis (PFGE) and multi-locus sequence typing (MLST) were used to determine the genotypes and homology of these isolates. Plasmids were analyzed by PFGE and conjugation experiments. The outer membrane proteins were examined by PCR and SDS-PAGE. All of the isolates were revealed to be resistant to the third and fourth generation cephalosporins and piperacillin-tazobactam. Tigecycline, colistin, levofloxacin and amikacin were successful against all of the isolates. The antibiotic resistance rates of aztreonam, gentamicin, trimethoprim-sulfamethoxazole and fosfomycin were 13.51, 48.64, 78.38 and $86.49 \%$, respectively. Of the 37 cases, 25 isolates

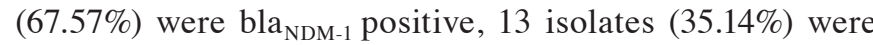
bla $_{\text {IMP-4 }}$ positive and 1 isolate $(2.70 \%)$ was bla IMP-8 $_{\text {I }}$ positive. Two isolates carried both bla $_{\mathrm{NDM}-1}$ and bla $\mathrm{IMP}-4_{4}$. The isolate carrying 2-4 plasmids and bla $\mathrm{NDM}-1_{1}$ and bla $\mathrm{IMP}-4_{4}$ was transferable between strains. SDS-PAGE data indicated that outer membrane proteins remained present. PFGE revealed 7 distinct clusters,
\end{abstract}

Correspondence to: $\mathrm{Dr}$ Chunhong Shao, Department of Microbiology, Clinical Laboratory, Provincial Hospital Affiliated to Shandong University, 324 Jingwu Road, Jinan, Shandong 250021, P.R. China

E-mail: lcsch@163.com

*Contributed equally

Key words: Klebsiella pneumoniae, carbapenem-resistance, infection of newborns, NDM-1, IMP-4 and MLST reported the presence of ST20, ST17, ST54, ST705 and ST290 sequences, which indicated that there was clone and plasmid spread between newborns. The main resistance mechanism of carbapenem-resistant Klebsiella pneumoniae was that the isolates expressed the carbapenemase resistance of bla $\mathrm{NDM}-1_{1}$ and bla $\mathrm{IMP}-4$ genes. The current study indicates that early detection of these genes may be helpful in infection prevention and control.

\section{Introduction}

Klebsiella pneumoniae is an important pathogen of community-acquired and nosocomial neonatal infections, with mortality rates varying between 18 and $68 \%(1,2)$. Carbapenem is the most effective drug used to treat infection with gram-negative bacteria, due to its marked antibacterial activity and stability in response to $\beta$-lactamases (3). However, the inappropriate use of carbapenem has accelerated the emergence of resistant strains in recent years (4). Infections associated with carbapenem-resistant $K$. pneumoniae are a public health problem globally due to their transmission mechanism and the limited therapeutic options available (5).

The most prevalent carbapenemase genes expressed by Enterobacteriaceae are in Ambler classes A and B, particularly $b l a_{\mathrm{KPC}}, b l a_{\mathrm{IMP}}$ and $b l a_{\mathrm{NDM}}(6)$. Steinmann et al (7) described a hospital outbreak caused by $K$. pneumoniae carbapenemase (KPC)-2-producing K. pneumoniae in Germany between July 2010 and January 2011 that resulted in the death of four people. Similarly, in March 2011, a New Delhi Metallo- $\beta$-lactamase (NDM)-1-producing Escherichia coli strain was isolated in Hong Kong, China (8).

The prevalence of carbapenem-resistant Enterobacteriaceae is increasing in mainland China, and has caused a number of nosocomial outbreaks. In 2012, Hu et al (9) reported an outbreak of 77 KPC-2-producing Enterobacteriaceae in Shanghai Huashan Hospital, China. Finally, in 2015, Chen et al (10) reported Enterobacteriaceae co-expressing bla $\mathrm{NDM}-1_{1}$ and bla $_{\mathrm{IMP}-4}$ in China. The prevalence of carbapenem-resistant Enterobacteriaceae is a current challenge during treatment and infection control in hospitals; fortunately, neonatal cases are typically rare. 
Table I. Primers used in the present study.

\begin{tabular}{|c|c|c|}
\hline Primer & Forward (5'-3') & Reverse $\left(5^{\prime}-3^{\prime}\right)$ \\
\hline KPC & ATTGGCTAAAGGGAAACACGACC & GTAGACGGCCAACACAAT \\
\hline SME & AGATAGTAAATTTTATAG & СТСТАACGCTAATAG \\
\hline IMIF & ATAGCCATCCTTGTTTAGCTC & TCTGCGATTACTTTATCCTC \\
\hline NMC & GCATTGATATACCTTTAGCAGAGA & CGGTGATAAAATCACACTGAGCATA \\
\hline GES & GTTTTGCAATGTGCTCAACG & TGCCATAGCAATAGGCGTAG \\
\hline IMP-1 & TGAGCAAGTTATCTGTATTC & TTAGTTGCTTGGTTTTGATG \\
\hline VIM-1 & TTATGGAGCAGCAACCGATGT & CAAAAGTCCCGCTCCAACGA \\
\hline GIM-1 & AGAACCTTGACCGAACGCAG & ACTCATGACTCCTCACGAGG \\
\hline SIM-1 & TACAAGGGATTCGGCATCG & TAATGGCCTGTTCCCATGTG \\
\hline SPM-1 & СCTACAATCTAACGGCGACC & TCGCCGTGTCCAGGTATAAC \\
\hline NDM-1 & ATTAGCCGCTGCATTGAT & GGCATGTCGAGATAGGAAGT \\
\hline OXA & ACACAATACATATCAACTTCGC & AGTGTGTTTAGAATGGTGATC \\
\hline CTX-M-cons & TTTGCGATGTGCAGTACCAGTAA & CGATATCGTTGGTGGTGCCATA \\
\hline CTX-M-1 & GGTTAAAAAATCACTGCGTC & TTACAAACCGTYGGTGACGA \\
\hline CTX-M-2 & ATGATGACTCAGAGCATTCGCCGC & TCAGAAACCGTGGGTTACGATTTT \\
\hline CTX-M-9 & GTGACAAAGAGAGTGCAACGG & ATGATTCTCGCCGCTGAAGCC \\
\hline CTX-M-15 & CACACGTGGAATTTAGGGACT & GCCGTCTAAGGCGATAAACA \\
\hline TEM & ACATGGGGGATCATGTAACT & GACAGTTACAATGCTTACT \\
\hline SHV & ATGCGTTATATTCGCCTGTG & AGCGTTGCCAGTGCTCGATG \\
\hline MOX & GCTGCTCAAGGAGCACAGGAT & CACATTGACATAGGTGTGGTGC \\
\hline FOX & AACATGGGGTATCAGGGAGATG & CAAAGCGCGTAACCGGATTGG \\
\hline DHA & AACTTTCACAGGTGTGCTGGGT & CCGTACGCATACTGGCTTTGC \\
\hline CTT & TGGCCAGAACTGACAGGCAAA & TTTCTCCTGAACGTGGCTGGC \\
\hline $\mathrm{EBC}$ & TCGGTAAAGCCGATGTTGCGG & СTTCCACTGCGGCTGCCAGTT \\
\hline OMPK35 & ATGATGAAGCGCAATATTCTGGCAGTGG & TGGGCTTTGTCGCCATTGCCGTCA \\
\hline OMPK36 & ATGAAAGTTAAAGTACTGTCCCTC & GCCGGTATCTCTACCGACGAC \\
\hline
\end{tabular}

In the present study, patients who were carbapenem-resistant Klebsiella pneumoniae-positive in the neonatal, pediatric intensive care and cardiac intensive care units (ICUs) were enrolled, between April 2011 and October 2013. In order to better understand infection control, the antibiotic resistance of the strains, and homogeneity and transmission mechanisms of the resistance genes, were investigated.

\section{Materials and methods}

Collection of isolates and susceptibility testing. In the current study, 37 carbapenem-resistant K. pneumoniae samples were collected in Shandong Provincial Hospital, China, between April 2011 and October 2013. Of these, 31 isolates were from the neonatal unit, 5 were from the pediatric ICU and 1 was from the cardiac ICU. All isolates were identified using a VITEK-2 compact 60 system (bioMérieux, Marcy l'Etoile, France). It is of note that 21 of these $K$. pneumoniae isolates carried with NDM-1 have been described in a previous article (11). The novel isolates were Kpn1, 3-8, 12, 14-18, 21, 24 and 39. Antibiotic susceptibility testing was performed by the agar dilution method (12). All antibiotics, except tigecycline and colisin, were administered according to the approved standard of the Clinical and Laboratory Standards Institute 2014 guidelines (13). The minimum inhibitory concentrations (MICs) of meropenem, imipenem, ertapenem, tigecycline, colisin and fosfomycin were determined by an Etest (bioMérieux). The 2014 European
Committee on Antimicrobial Susceptibility Testing breakpoint (www.eucast.org/clinical_breakpoint) was used to determine resistance in colistin and tigectcline. All isolates were screened for carbapenemase production using the modified Hodge test (MHT) and imipenem-ethylenediaminetetraacetic acid (EDTA) double-disc synergistic test (14). E. coli ATCC25922 (American Type Culture Collection Center, Manassas, VA, USA) acted as the control.

Polymerase chain reaction (PCR) and DNA sequence analysis of drug resistance genes. Samples were screened for the presence of carbapenem resistance genes (blaKPC, blaSME, blaIMI/blaNMC, blaGES, blaIMP, blaVIM, blaGIM, blaSIM-1, blaSPM, blaNDM-1 and blaOXA), common extended-spectrum $\beta$-lactamase (ESBL) genes (blaCTX-M, blaTEM and blaSHV) and AmpC $\beta$-lactamase (AMPC) genes (blaMOX, bla FOX, blaDHA, blaCIT and blaEBC) in all 37 strains using PCR and previously described primers (15-18). The primers used are listed in Table I. The genome used as a template was extracted using a Rapid Bacterial Genomic DNA Isolation Kit (Sangon Biotech Co., Ltd., Shanghai, Chin). PCR was performed according to the manufacturer's instruction of the PCR (Taq) kit (B532501-0100; Sangon Biotech Co., Ltd.). The reaction was performed using the following thermal cycling conditions: 1 Cycle at $94^{\circ} \mathrm{C}$ for $5 \mathrm{~min}$; 30 cycles at $94^{\circ} \mathrm{C}$ for $1 \mathrm{~min}, 55-58^{\circ} \mathrm{C}$ for $45 \mathrm{sec}$ and $72^{\circ} \mathrm{C}$ for $1 \mathrm{~min}$; and 1 cycle at $72^{\circ} \mathrm{C}$ for $10 \mathrm{~min}$. The results of PCR were screened 


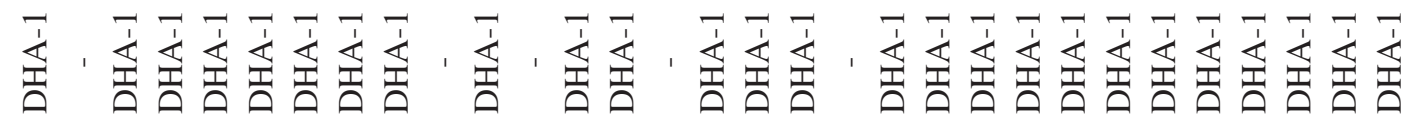

オ

$\sum_{1} \sum \sum \sum \sum \sum \sum \sum \sum \sum \sum \sum \sum \sum \sum \sum \sum \sum \sum \sum \sum \sum \sum \sum \sum \sum \sum \sum \sum \sum \sum$

离离离离离离离离离离离离离离离离离离离离离离离

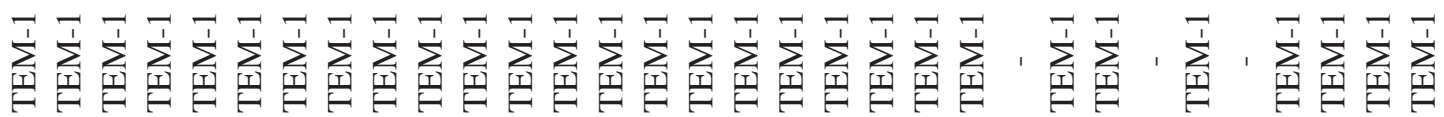

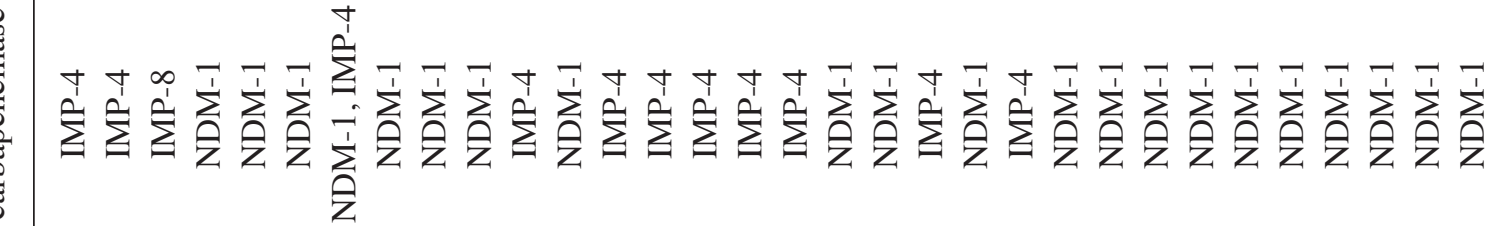

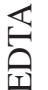

产

멍

约

क्ष 苛

山

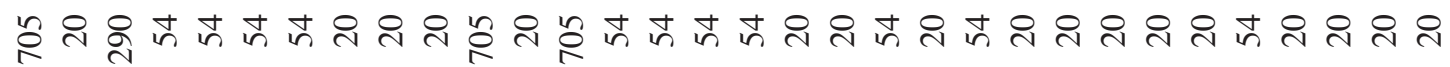

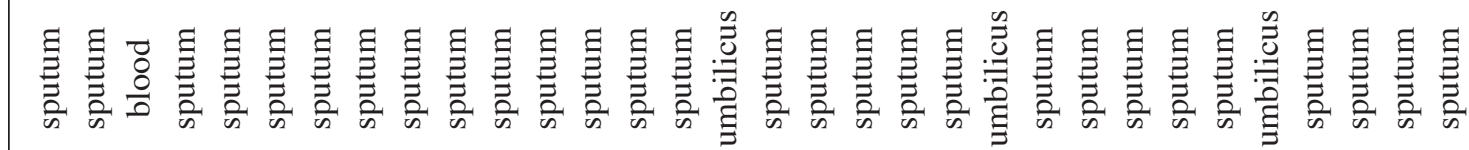

$m$ m

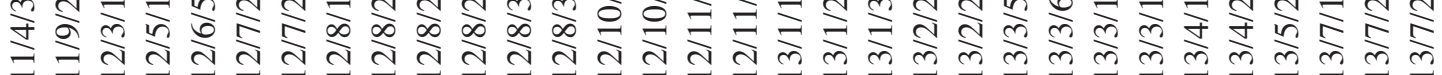

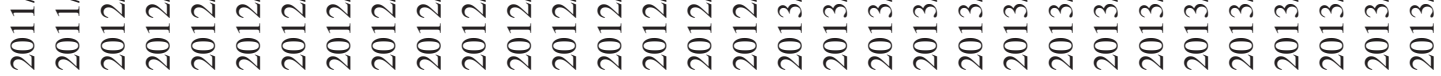

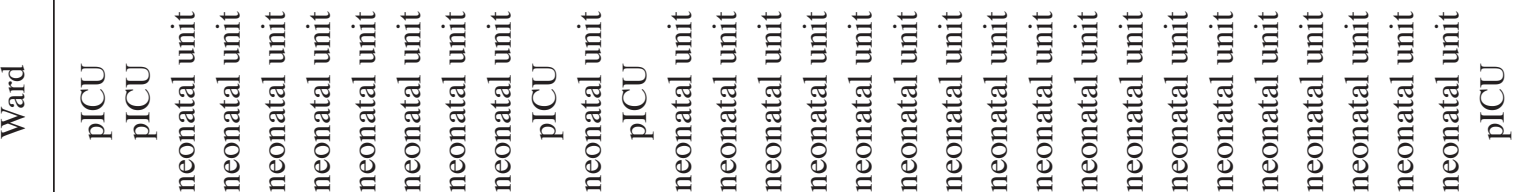

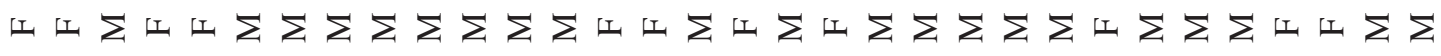

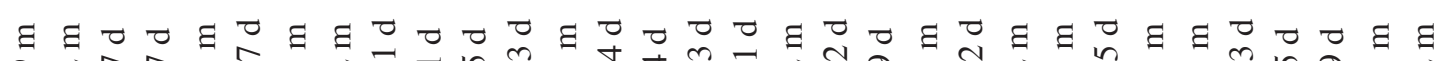
acr 
by electrophoresis on a $1 \%$ agarose gel and the results were sequenced by Sangon Biotech Co., Ltd.

Resistance gene transfer experiments. Transfer experiments were performed using azide-resistant $E$. coli J53 (donated by Peking Union Medical College Hospital, Beijing, China) as the recipient strain. Overnight single colonies of donor and acceptor strains on MacConkey agar plates were inoculated into $5 \mathrm{ml}$ nutrient broth containing imipenem $(0.5 \mu \mathrm{g} / \mathrm{ml})$ or sodium azide $(200 \mu \mathrm{g} / \mathrm{ml})$. These were incubated at $37^{\circ} \mathrm{C}$ for $8 \mathrm{~h}$. Cultures of the donor strain $(10 \mu \mathrm{l})$ and recipient strain $(20 \mu \mathrm{l})$ were mixed with $10 \mathrm{ml}$ fresh Mueller-Hinton broth and incubated for $24 \mathrm{~h}$ at $35^{\circ} \mathrm{C}$. The mixture was then inoculated onto MacConkey agar plates containing sodium azide $(200 \mu \mathrm{g} / \mathrm{ml})$ and imipenem $(0.5 \mu \mathrm{g} / \mathrm{ml})$ for $24 \mathrm{~h}$ at $35^{\circ} \mathrm{C}$. Conjugation was confirmed with a VITEK-2 compact system for drug sensitivity testing. The presence of carbapenemase was confirmed by MHT, imipenem-EDTA double-disc synergistic test and PCR analysis.

Pulse-field gel electrophoresis (PFGE) and plasmid analysis. An overnight bacterial culture was suspended in cell suspension buffer [100 mM EDTA, $100 \mathrm{mM}$ Tris- $\mathrm{HCl}$ ( $\mathrm{pH}$ 8.0)] and adjusted to an optical density of 4.0 at a wavelength of $600 \mathrm{~nm}$. The suspension was mixed with equal volumes of low-melting agarose in Tris-EDTA (TE) buffer in a $2 \%$ solution of low-melting-temperature agarose in TE buffer [1 mM EDTA, $10 \mathrm{mM}$ Tris-HCl (pH 8.0)]. Following cooling, the agarose sections were incubated for $4 \mathrm{~h}$ at $54^{\circ} \mathrm{C}$ in cell lysis buffer [50 mM Tris- $\mathrm{HCl}, 50 \mathrm{mM}$ EDTA $(\mathrm{pH}$ 8.0), $0.01 \mathrm{~g} / \mathrm{ml} \mathrm{N}$-lauroyl-sarcosine, sodium salt, $0.1 \mathrm{mg} / \mathrm{ml}$ proteinase $\mathrm{K}$ ]. These were then washed thoroughly with $\mathrm{TE}$ buffer and digested overnight with XbaI restriction endonuclease (Takara Bio, Inc., Otsu, Japan). DNA separation was performed in $0.5 \mathrm{X}$ Tris/borate/EDTA (TBE) buffer in a PFGE system (CHEF Mapper; Bio-Rad Laboratories, Inc., Hercules, CA, USA) at $14^{\circ} \mathrm{C}$, using a voltage of $6 \mathrm{~V} / \mathrm{cm}$, a switch angle of $120^{\circ}$ and a switch ramp of 4-40 sec for 21 h. The Salmonella enterica serotype Braenderup H9812 (Peking Union Medical College Hospital) was used as a marker for PFGE. The restriction patterns were analyzed and interpreted in accordance with the protocol implemented by Tenover et al (19).

The plasmid number and size of the 37 isolates, and their transconjugants, were analyzed by S1-PFGE. In summary, the gels embedded with bacterial DNA were digested with proteinase $\mathrm{K}$ and $\mathrm{S} 1$ nuclease. DNA separation was performed in $0.5 \mathrm{X}$ TBE buffer in the above PFGE system at $14^{\circ} \mathrm{C}$ with a voltage of $6 \mathrm{~V} / \mathrm{cm}$, a switch angle of $120^{\circ}$ and a switch ramp of 2.16-63.8 sec for 20 h. S. enterica $\mathrm{H} 9812$ fragment, digested by XbaI enzyme, was used as a molecular weight standard. Subsequent to PFGE, the DNA products of the positive transconjugants were recovered and used as templates to amplify the carbapenem-resistance genes. The PCR reaction conditions and primers used are the same as described above. The PCR products were sequenced and searched on GenBank (www.ncbi.nlm.nih.gov/genbank).

Multilocus sequence typing (MLST). MLST was performed on $K$. pneumoniae using 7 conserved housekeeping genes 


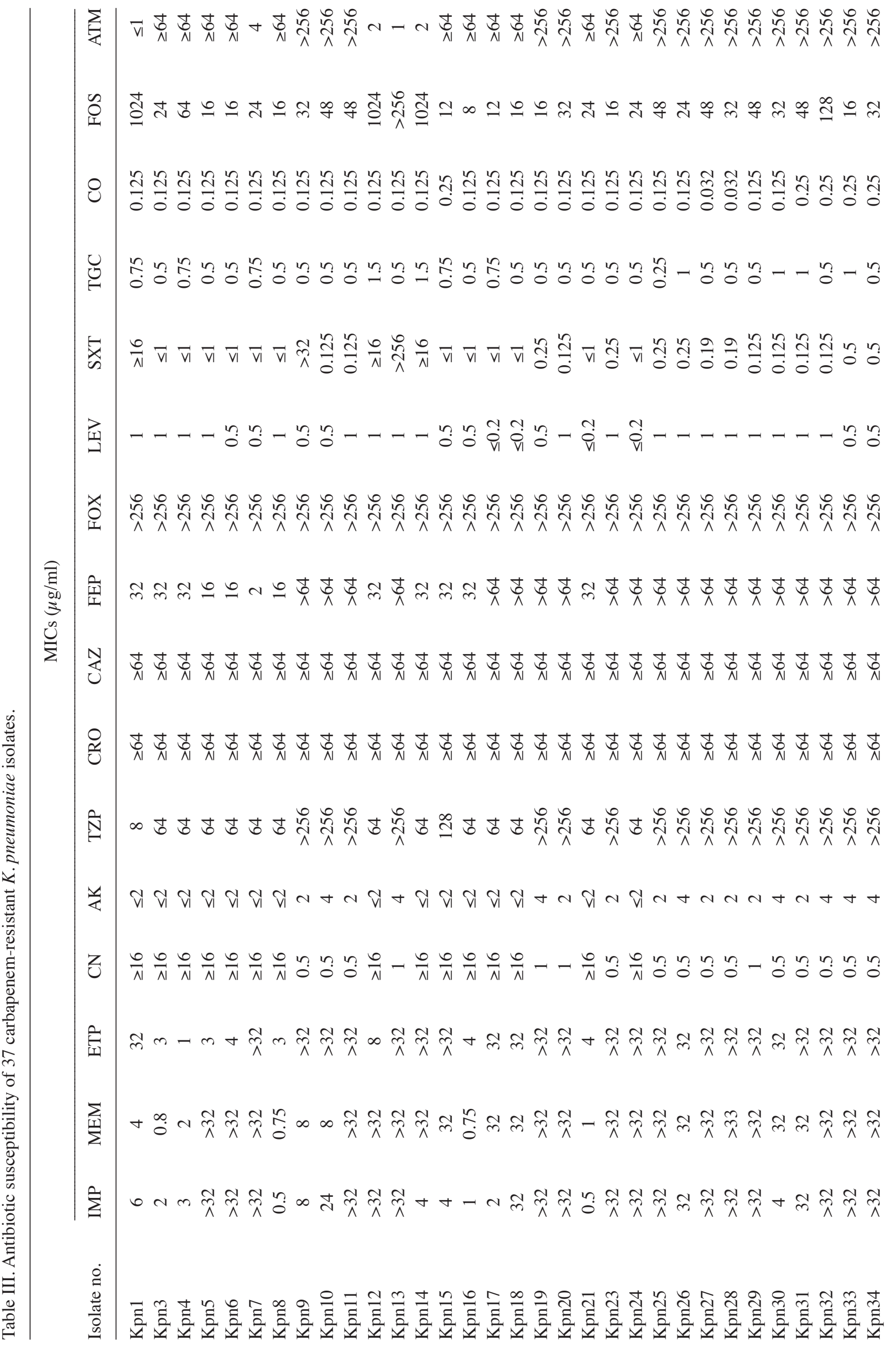


(gapA, infB, $m d h, p g i, p h o E, r p o B$ and tonB) according to protocols available on the MLST Pasteur website (bigsdb.pasteur.fr/klebsiella/primers_used.html).

Analysis of outer membrane proteins (OMPs). The OMP genes were screened in all clinical isolates with previously described primers, using PCR (20). The primers used are listed in Table I. The reaction used the following thermal cycling conditions: 1 Cycle at $94^{\circ} \mathrm{C}$ for $5 \mathrm{~min} ; 30$ cycles at $94^{\circ} \mathrm{C}$ for $45 \mathrm{sec}, 55^{\circ} \mathrm{C}$ for $45 \mathrm{sec}$ and $72^{\circ} \mathrm{C}$ for $1 \mathrm{~min}$; and 1 cycle at $72^{\circ} \mathrm{C}$ for $10 \mathrm{~min}$. The results of PCR were screened by electrophoresis on a $1 \%$ agarose gel. The PCR negative isolates were investigated for alterations in the OMPs by SDS-PAGE, as previously described (21).

\section{Results}

Clinical and epidemiological characteristics of 37 isolates. Between April 2011 and October 2013, 37 carbapenem-resistant $K$. pneumoniae samples were isolated from neonatal inpatients in Shandong Provincial Hospital in Jinan, China. Of these, 32 isolates were from sputum specimens, 3 were from umbilical secretions and 2 were from blood (Table II). As indicated, 21 of these K. pneumoniae isolates carried with NDM-1 have been described in a previous article (11). Of these samples, 31 were isolated from patients in the neonatal department, 5 were from the pediatric ICU and 1 was from a patient in the cardiac ICU. These patients included 23 males and 14 females. The 31 patients from the neonatal ward were all premature, with a low birth weight and neonatal pneumonia. A total of 5 infant patients had an intrauterine infection at birth. All patients were initially treated with various antibiotics, including cloxacillin, cefuroxime, and cefotetan. A total of 8 patients were treated with imipenem or meropenem. The cardiac ICU patient had a respiratory tract infection and fever 5 days after their cardiac operation. The prognosis of this cohort was poor; 2 patients succumbed to bloodstream infection.

Susceptibility results. Drug sensitivity was tested with the standard agar dilution method (Table III). These features in 21 previously described $K$. pneumoniae isolates are included in this analysis (11). All 37 isolates were resistant to penicillin, piperacillin-tazobactam, cephalosporins and their composite agents including the enzyme inhibitors cefoxitin and carbapenem. The percentage of the number of bacteria senstive to antibiotics divided by total strains, the sensitivity rate, to tigecycline, levofloxacin, amikacin and polymyxin were all $100 \%$. The sensitivity rates of aztreonam, gentamicin, trimethoprim-sulfamethoxazole and fosfomycin were 13.51, 48.64, 78.38 and $86.49 \%$, respectively.

The 37 K. pneumoniae samples displayed different degrees of resistance to carbapenems. Kpn4 was sensitive to ertapenem, with intermediate sensitivity to imipenem and meropenem. Of the 7 strains with mild sensitivity to ertapenem, 4 strains (Kpn16,21,3 and 8) were sensitive to imipenem and meropenem, 2 strains (Kpn5 and 6) were markedly resistant to imipenem and meropenem and Kpn39 was resistant to imipenem, with intermediate resistance to meropenem. The 19 isolates all had high resistance to the three carbapenem antibiotics; the MICs of the three carbapenems were $\geq 32 \mu \mathrm{g} / \mathrm{ml}$. 


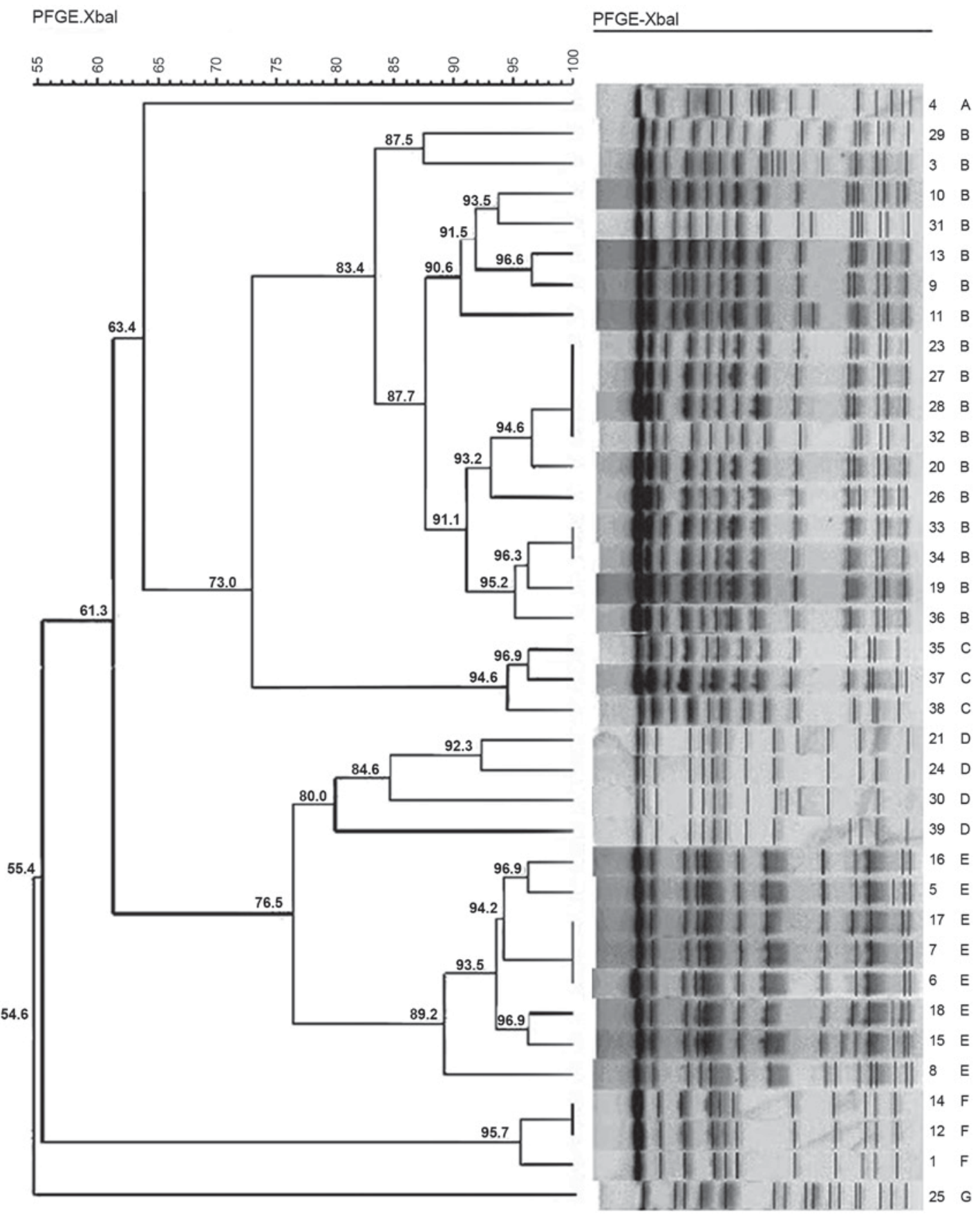

Figure 1. Dendrogram analysis. Dendrogram generated with the Fingerprinting II Informatix software package, demonstrating the relatedness of fingerprints (XbaI-PFGE) for 37 carbapenem-resistant $K$. pneumoniae strains. The phylogenetic tree was constructed using the Dice coefficient and UPGMA clustering. A genetic similarity index scale is shown in the left of the dendrogram. PFGE types and strain number are included along each PFGE lane. PFGE, pulse-field gel electrophoresis.

Resistance characterization and resistance genes. The 37 carbapenem-resistant $K$. pneumoniae, including those from our previous study, all showed positive results in the EDTA synergistic test, and 7 attained positive or weakly positive results in the MHT, at a rate of $18.92 \%$ (11). Metallo- $\beta$-lactamases (MBL) were confirmed to be present in all isolates through sequencing of the PCR products. Of these, 23 isolates expressed only $b l a_{\mathrm{NDM}-1}$, and 11 isolates expressed only $b l a_{\mathrm{IMP}-4}$. Kpn4, isolated from blood, expressed only $b l a_{\mathrm{IMP}-8}$, and 2 isolates simultaneously expressed $b l a_{\mathrm{NDM}-1}$ and $b l a_{\mathrm{IMP}-4}(11)$. In addition to MBL, other types of $\beta$-lactamase were examined, including ESBLs and AMPCs. The distribution of the resistance genes in these strains is reported in Table II. The ESBL genes were determined to be $b l a_{\mathrm{CTX}-\mathrm{M}-15}, b l a_{\mathrm{CTX}-\mathrm{M}-14}$ and $b l a_{\mathrm{TEM}-1}$ in $24(64.87 \%), 13(35.14 \%)$ and $34(91.89 \%)$ isolates, respectively. Genotyping of the AMPC genes confirmed the presence of $b l a_{\mathrm{DHA}-1}$ in 28 (75.68\%) isolates.

Characterization of OMPs. In our previous study, the outer membrane protein genes OmpK35 and OmpK36 were detected in $21 \mathrm{~K}$. pneumoniae isolates expressing NDM-1 (Kpn9, Kpn10, Kpn11, Kpn13, Kpn19, Kpn20, Kpn23 and Kpn25-38) (11). The remaining 16 strains revealed no loss of OmpK35 and OmpK36 at the gene or the protein level. 
Transfer of carbapenem resistance. A total of 28 transconjugants were obtained from 37 isolates by transfer experiments, with a success rate of $75.68 \%$. Of these, 20 transconjugants were obtained from 23 isolates encoding bla $\mathrm{NDM}_{\mathrm{ND}-1}$ and 8 transconjugants were obtained from 11 isolates encoding $\operatorname{bla}_{\mathrm{IMP}-4}(11)$. The transfer experiment failed in 2 isolates simultaneously encoding $\mathrm{bla}_{\mathrm{NDM}-1}$ and $\mathrm{bla}_{\mathrm{IMP}-4}$. All transconjugants were confirmed to have the same biochemical spectrum as E. coli strain J53, using the VITEK-2 compact system. All transconjugants demonstrated similar carbapenem susceptibility to donor strains Table IV. Compared with E. coli strain J53, the MIC of imipenem, meropenem and etarpenem increased 8- to 64-fold, 32- to 512-fold and 256- to 2,048-fold, respectively. The MIC of piperacillin tazobactam, ceftazidime, Cefatriaxone, cefoxitin and cefepime increased 8- to 2,048-fold.

Homology analyzed by PFGE and MLST. MLST revealed that the 37 isolates had 5 different sequence types: ST17, ST20, ST54, ST705 and ST290 (Table III) (11). The ST20 and ST54 sequences represented the majority of the clones. PFGE revealed 7 distinct clusters amongst the 37 isolates (Fig. 1). The 3 strains from the umbilical cord all had ST54 sequences, while two strains (Kpn4 and Kpn9) isolated from blood had ST54 and ST290 sequences. Cluster B included 17 isolates, all of which had ST20 sequences. The 12 isolates with ST54 sequences were divided into clusters D and E. A cluster was defined as strains with homology $>80 \%$.There were 5 strains isolated from the pediatric ICU, 3 of which had ST705 sequences and were allocated into cluster F. The Kpn35, Kpn37 and Kpn38 samples belonged to ST17 and the same cluster E in PFGE (11).

Plasmid profiling. The plasmid number and size of all isolates were analyzed by S1-PFGE, which revealed that these isolates contained 2-4 plasmids. As the primary sequences, ST20- and ST54-encoding isolates contained 2 or 3 plasmids, but their transconjugants only had 1 plasmid. The sizes of the plasmid from ST20- and ST54-encoding bacteria were $336 \mathrm{~kb}$ and $55 \mathrm{~kb}$, respectively. The $\mathrm{bla}_{\mathrm{NDM}-1}$ and $\mathrm{bla}_{\mathrm{IMP}-4}$ were located in the $336 \mathrm{~kb}$ plasmid and $55 \mathrm{~kb}$ plasmid, respectively, as determined by PCR and sequencing.

\section{Discussion}

The standard method of treatment for serious infections caused by ESBL-producing Enterobacteriaceae is carbapenem antibiotics, but drug resistance is a critical problem (22). The present study demonstrated that carbapenem-resistant $K$. pneumoniae primarily manifested in infants within the neonatal unit. Premature birth, low birth weight, low immunity and intrauterine infections are the primary risk factors of nosocomial infection (23). The widespread inappropriate use of carbapenem and cephalosporins is an additional important risk factor (4).

In the current study, all patients were treated with cephalosporins within 2 weeks of admission, and 8 infants were prescribed carbapenem. The use of a ventilator may increase the risk of infection by carbapenem-resistant $K$. pneumoniae, however 10 patients used a ventilator in the present study. Antibiotic sensitivity testing revealed that carbapenem-resistant
K. pneumoniae was resistant to most antibiotics. Only a few antibiotics, including aminoglycosides, fluoroquinolones, colistin, tigecycline and fosfomycin, were effective in vitro.

There is currently no effective drug available to treat carbapenem-resistant Enterobacteriaceae in neonates due to the limited range of drugs available. This it because some antibiotics, such as aminoglycosides, quinolones and tetracyclines, can not be used in pediatric patients because of their side effects. Therefore, infection by carbapenem-resistant Enterobacteriaceae has a very high mortality rate of $\sim 70 \%$ in patients with bacteremia (24). In the current study, the prognosis of all neonates was poor; 2 children succumbed to bloodstream infections.

The outbreak of $21 \mathrm{~K}$. pneumoniae isolates expressing NDM-1 were reported in a previous article (11). In addition, the present study described an outbreak of the same clone (ST705) was identified in the pediatric ICU. This strain was resistant to all beta-lactam antibiotics except for azteronam. The current results confirmed that this resistance was induced by the metalloenzyme bla $\mathrm{IMP}-4_{4}$, belonging to the Ambler class B of carbapenemases. This strain is able to hydrolyze penicillins, cephalosporins and carbapenems, but not aztreonam. In the neonatal ward, there were 2 strains of carbapenem-resistant $K$. pneumoniae: ST20 and ST54. The former carried $b a_{\mathrm{NDM}-1}$ and was responsible for an outbreak between August 2012 and July 2013. In comparing the housekeeping genes of ST20 and ST17, only the infB allele was reported to differ, with a base variant at 279 (T-C). This suggested that these 2 sequences are highly homologous. K. pneumoniae of the ST54 strain was identified with 5 isolates expressing only bla $_{\mathrm{NDM}-1}, 7$ isolates expressing only $\mathrm{bla}_{\mathrm{IMP}-4}$, and Kpn8 expressing $\mathrm{bla}_{\mathrm{NDM}-1}$ and $\mathrm{bla}_{\mathrm{IMP}-4}$. The ST54 strains expressing bla $_{\mathrm{NDM}-1}$ were present in samples between May 2012 and July 2012, and the ST54 strains expressing bla $_{\text {IMP-4 }}$ were prevalent in samples between 2011 and 2013 . The outbreak described in the present study was due to the ST54 strain, expressing bla $\mathrm{NDM}-1_{1}$. Critically, 2 isolates expressed both bla $\mathrm{NDM}_{\mathrm{N}-1}$ and $\mathrm{bla}_{\mathrm{IMP}-4}$.

The production of carbapenemase was the primary mechanism by which Enterobacteriaceae bacteria attained resistance to carbapenem antibiotics. Carbapenemase-producing Enterobacteriaceae induce high mortality and are easily spread by acquiring carbapenemases from different species $(25,26)$. In the present studied population, the most common genes expressed were $\mathrm{bla}_{\mathrm{NDM}-1}$ and bla $\mathrm{IMP}-4$. Plasmid analysis proved that NDM-1 and IMP-4 were located in $336 \mathrm{~kb}$ and $55 \mathrm{~kb}$ plasmids, respectively. A transformation test revealed that these are easily transferred between different strains, and may cause spread between bacteria. Previous studies have reported that infection in neonates is caused by $\mathrm{bla}_{\mathrm{CTX}-\mathrm{M}-15}$-expressing $\mathrm{K}$. pneumoniae in the strains ST20 and ST17 in Spain and Canada $(27,28)$. The outbreak of carbapenem-resistant $K$. pneumoniae in causing neonatal infection therefore requires additional study.

Previous studies have suggested that the overexpression of ESBLs and AMPC combined with missing outer membrane proteins have an important role in the drug resistance of K. pneumoniae $(29,30)$. Currently, the main known outer membrane proteins in K. pneumoniae include OmpK37, OmpK35 and OmpK36. Of these, OmpK35 and OmpK36 have crucial roles in regulating drug penetration (11). While previous 


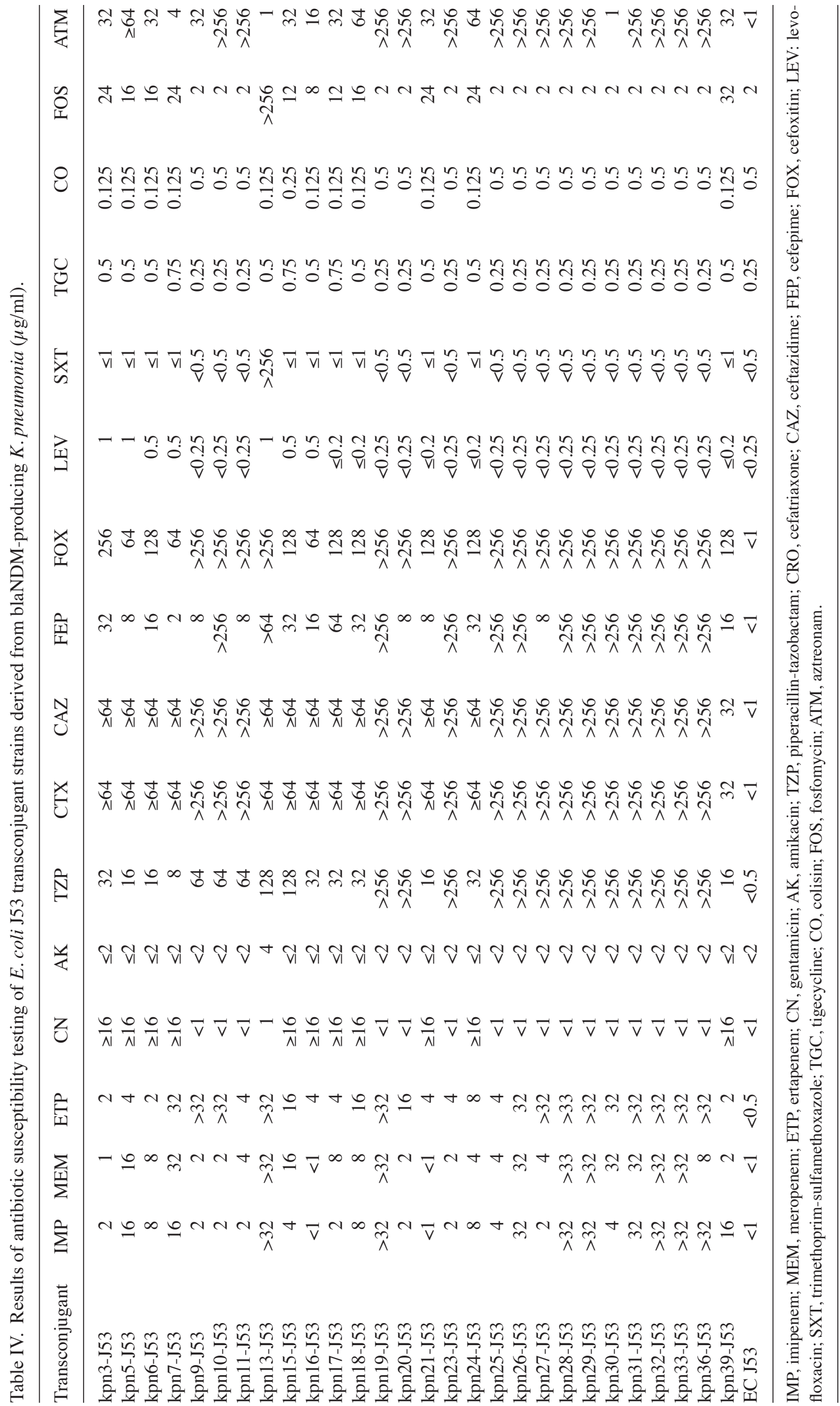


PCR amplification reported gene deletion of OmpK35 in kpn15 and kpn38, SDS-PAGE did not confirm the loss of OmpK35. This suggested that detection of the membrane protein required a combination of PCR and SDS-PAGE methods. Furthermore, it indicated that the resistance to carbapenems by $K$. pneumoniae was not associated with the outer membrane protein. Carbapenem-resistant $K$. pneumoniae expressed the carbapenemases gene, and ESBLs and AMPC, also permitting resistance to beta-lactam drugs, suggesting the presence of multi-drug resistance genes.

In conclusion, carbapenem-resistant $K$. pneumoniae causes neonatal infection, which may be due to simultaneously expressed multiple drug resistance genes. In the present study, bla ${ }_{\mathrm{NDM}-1}$ and $\mathrm{bla}_{\mathrm{IMP}-4}$ were the predominating resistance genes. Minimizing spread is a critical clinical challenge. carbapenem-resistant Klebsiella pneumoniae is a severe threat to the healthcare system, and calls for stringent standard infection control practices in healthcare settings, in addition to the reasonable use of antibiotics.

\section{Acknowledgements}

The present study was supported by the National Natural Science Foundation of China (grant no. 81401696) and the Shandong Province Science and Technology Development Program of China (grant no. 2012G0021844).

\section{References}

1. Saleem AF, Qamar FN, Shahzad H, Qadir M and Zaidi AK Trends in antibiotic susceptibility and incidence of late-onset Klebsiella pneumoniae neonatal sepsis over a six-year period in a neonatal intensive care unit in Karachi, Pakistan. Int J Infect Dis 17: e961-e965, 2013.

2. Schwaber MJ, Lev B, Israeli A, Solter E, Smollan G, Rubinovitch B, Shalit I and Carmeli Y; Israel Carbapenem-Resistant Enterobacteriaceae Working Group: Containment of a country-wide outbreak of carbapenem-resistant Klebsiella pneumoniae in Israeli hospitals via a nationally implemented intervention. Clin Infect Dis 52: 848-855, 2011.

3. Nicolau DP: Carbapenems: A potent class of antibiotics. Expert Opin Pharmacother 9: 23-37, 2008.

4. Ulu AC, Kurtaran B, Inal AS, Kömür S, Kibar F, Çiçekdemir HY, Bozkurt S, Gürel D, Kılıç F, Yaman A, et al: Risk factors of carbapenem-resistant Klebsiella pneumoniae infection: A serious threat in ICUs. Med Sci Monit 21: 219-224, 2015.

5. Kontopidou F, Giamarellou H, Katerelos P, Maragos A, Kioumis I, Trikka-Graphakos E, Valakis C and Maltezou HC; Group for the Study of KPC-producing Klebsiella pneumoniae infections in intensive care units: Infections caused by carbapenem-resistant Klebsiella pneumoniae among patients in intensive care units in Greece: A multi-centre study on clinical outcome and therapeutic options. Clin Microbiol Infect 20: O117-O123, 2014.

6. Diene SM and Rolain JM: Carbapenemase genes and genetic platforms in Gram-negative bacilli: Enterobacteriaceae, Pseudomonas and Acinetobacter species. Clin Microbiol Infect 20 831-838, 2014

7. Steinmann J, Kaase M, Gatermann S, Popp W, Steinmann E, Damman M, Paul A, Saner F, Buer J and Rath P: Outbreak due to a Klebsiella pneumoniae strain harbouring KPC-2 and VIM-1 in a German university hospital, July 2010 to January 2011. Euro Surveill 16: 19944, 2011.

8. Ho PL, Lo WU, Yeung MK, Lin CH, Chow KH, Ang I, Tong AH, Bao JY, Lok S and Lo JY: Complete sequencing of pNDM-HK encoding NDM-1carbapenemase from a multidrug-resistant Escherichia coli strain isolated in Hong Kong. PLoS One 6: e17989, 2011

9. Hu F, Chen S, Xu X, Guo Y, Liu Y, Zhu D and Zhang Y: Emergence of carbapenem-resistant clinical Enterobacteriaceae isolates from a teaching hospital in Shanghai, China. J Med Microbiol 61: 132-136, 2012.
10. Chen Z, Wang Y, Tian L, Zhu X, Li L, Zhang B, Yan S and Sun Z: First Report in China of enterobacteriaceae clinical isolates coharboring blaNDM-1 and blaIMP-4 drug resistance genes. Microb Drug Resist 21: 167-170, 2015.

11. Jin Y, Shao C, Li J, Fan H, Bai Y and Wang Y: Outbreak of Multidrug Resistant NDM-1-Producing Klebsiella pneumoniae from a neonatal unit in Shandong Province, China. PLoS One 10: e0119571, 2015.

12. Clinical and Laboratory Standards Institute: Method for dilution antimicrobial susceptibility tests for bacteria that grow aerobically; approved standard-ninth edition, 2012, M07-A9. Wayne, PA:CLSI, 2012.

13. Clinical and Laboratory Standards Institute: Performance standards for antimicrobial susceptibility testing twentieth informational supplement, 2014, M100-S24. Wayne, PA:CLSI, 2014.

14. Lee K, Chong Y, Sllin HB, Kim YA, Yong D and Yum JH: Modified Hodge and EDTA disk synergy tests to screen metallo-beta-lactamase producing strains of Pseudomonas and Acinetobacter species. Clin Microbiol Infect 7: 88-91, 2001

15. Queenan AM and Bush K: Carbapenemases: The versatile beta-lactamases. Clin Microbiol Rev 20: 440-458, 2007.

16. Fang H, Ataker F, Hedin G and Dornbusch K: Molecular epidermiology of extended-spectrum beta-lactamases among Escherichia coli isolates collected in a Swedish hospital and its associated health care facilities from 2001 to 2006. J Clin Microbio 46: 707-712, 2008.

17. Pérez-Pérez FJ and Hanson ND: Detection of plasmid-mediated AmpC beta-lactamase genes in clinical isolates by using multiplex PCR. J Clin Microbio 40: 2153-2162, 2002.

18. Robicsek A, Strahilevitz J, Sahm DF, Jacoby GA and Hooper DC: qnr prevalence in ceftazidime-resistant enterobacteriaceae isolates from the United States. Antimicrob Agents Chemother 50: 2872-2874, 2006.

19. Tenover FC, Arbeit RD, Goering RV, Mickelsen PA, Murray BE, Persing DH and Swaminathan B: Interpreting chromosomal DNA restriction patterns produced by pulsed-field gel electrophoresis: Criteria for bacterial strain typing. J Clin Microbiol 33: 2233-2239, 1995

20. Doumith M, Ellington MJ, Livennore DM and Woodford N: Molecular mechanisms disrupting porin expression in ertapenem-resistant Klebsiella and Enterobacter spp. clinical isolates from the UK. J Antimicrob Chemother 63: 659-667, 2009.

21. Hernández-Allés S, Albertí S, Alvarez D, Doménech-Sánchez A, Martínez-Martínez L, Gil J, Tomás JM and Benedí VJ: Porin expression in clinical isolates of Klebsiella pneumoniae. Microbiology 145: 673-679, 1999.

22. Paterson DL: Recommendation for treatment of severe infection caused by Enterobacteriaceae producing extended-spectrum beta-lactamases (ESBLs). Clin Microbiol Infect 6: 460-463, 2000.

23. Ghotaslou R, Ghorashi Z and Nahaei MR: Klebsiella pneumoniae in neonatal sepsis: A 3-year-study in the pediatric hospital of Tabriz, Iran. Jpn J Infect Dis 60: 126-128, 2007.

24. Borer A, Saidel-Odes L, Riesenberg K, Eskira S, Peled N, Nativ R, Schlaeffer F and Sherf M: Attributable mortality rate for carbapenem-resistant Klebsiella pneumoniae bacteremia. Infect Control Hosp Epidemiol 30: 972-976, 2009.

25. Cherkaoui A, Emonet S, Renzi G, Riat A, Greub G and Schrenzel J: ESBL and carbapenemases in Enterobacteriaceae. Rev Med Suisse 10: 2142-2148, 2014 (In French).

26. Stock I: Infectious diseases caused by carbapenemase-producing Enterobacteriaceae - a particular challenge for antibacterial therapy. Med Monatsschr Pharm 37: 162-172; quiz 173-174, 2014 (In German).

27. Peirano G, Sang JH, Pitondo-Silva A, Laupland KB and Pitout JD: Molecular epidemiology of extended-spectrum- $\beta$-lactamase-pro ducing Klebsiella pneumoniae over a 10 year period in Calgary, Canada. J Antimicrob Chemother 67: 1114-1120, 2012.

28. Mavroidi A, Liakopoulos A, Gounaris A, Goudesidou M, Gaitana K, Miriagou V and Petinaki E: Successful control of a neonatal outbreak caused mainly by ST20 multidrug-resistant SHV-5-producing Klebsiella pneumoniae, Greece. BMC Pediatr 14: 105, 2014.

29. Delgado-Valverde M, Sojo-Dorado J, Pascual A and Rodríguez-Baño J: Clinical management of infections caused by multidrug-resistant Enterobacteriaceae. Ther Adv Infect Dis 1: 49-69, 2013.

30. Doménech-Sánchez A, Hernández-Allés S, Martínez-Martínez L, Benedí VJ and Albertí S: Identification and characterization of a new porin gene of Klebsiella pneumoniae: Its role in beta-lactam antibiotic resistance. J Bacteriol 181: 2726-2732, 1999. 